\title{
VASOPRESSINA E MORTE ENCEFÁLICA
}

\author{
ELIANE DE ARAUJO CINTRA*, JAYME ANTUNES MACIEL JUNIOR**, \\ SEBASTIÁO ARAÚJO ${ }^{* * *}$, MARGARET DE CASTRO ${ }^{* * * *}$, EDNA FREITAS MARTINS ${ }^{* * * * *}$
}

\begin{abstract}
RESUMO - A morte encefálica (ME) resulta numa perda completa dos mecanismos centrais de regulação da estabilidade hemodinâmica mesmo em pacientes com suporte adequado da ventilação, correção hidroeletrolítica e ácido-básica e suporte farmacológico convencional máximo da circulação. Acredita-se que a diminuição da vasopressina circulante influencia de maneira preponderante a estabilidade cardiocirculatória de pacientes com ME, sendo a sua administração exógena defendida por alguns autores no manuseio do potencial doador de órgãos. $\mathrm{O}$ artigo analisa e discute alguns estudos experimentais e clínicos relevantes em relação ao comportamento da vasopressina na ME e seu papel na manutenção da estabilidade cardiocirculatória, bem como sua potencial utilidade no manuseio destes pacientes. Desta análise concluímos que o comportamento da vasopressina na ME e o seu real valor na manutenção do potencial doador ainda não estão totalmente esclarecidos, necessitando de investigações futuras.
\end{abstract}

PALAVRAS-CHAVES: vasopressina, morte encefálica, estabilidade cardiocirculatória, potencial doador de órgãos.

\section{Vasopressin and brain death}

ABSTRACT - Brain death results in the breakdown of effective central regulatory mechanisms of cardiocirculatory stability, even in patients with artificial mechanical ventilation, correction of electrolytic and acid-basic disorders and maximal conventional pharmacological support of the circulation. Recent evidences have shown that the fall of vasopressin levels in the blood circulation significantly influences the cardiocirculatory stability of patients with brain death, and its exogenous administration is defended by many authors for the management of multiorgan donor patients. In this brief review we analyse and discuss some experimental and clinical relevant studies about the role of vasopressin in the control of cardiocirculatory stability in brain death, and its potential usefulness in the management of multiorgan donor. We conclude that the role of vasopressin in the pathophysiology of brain death and its usefulness as a pharmacological agent in the management of multiorgan donor are not well elucidated, deserving further investigations.

KEY WORDS: vasopressin, brain death, cardiocirculatory stability, and organ donor.

A morte encefálica ( $\mathrm{ME}$ ) é definida como condição na qual há perda irreversível das funções cerebrais, incluindo o tronco encefálico. As funções pulmonares e cardiocirculatórias podem ser mantidas através de medidas de suporte ( ventilação mecânica, drogas vasoativas, aquecimento artificial ) por tempos variáveis, após o aparecimento da $\mathrm{ME}^{1}$. A angiografia contrastada, nestes casos, quase invariavelmente demonstra o não preenchimento dos vasos cerebrais devido ao aumento da pressão intracraniana que ultrapassa a pressão arterial média ${ }^{2-4}$.

Faculdade de Ciências Médicas (FCM) - Universidade Estadual de Campinas (UNICAMP): *Enfermeira, mestranda do curso de pós graduação em Neurociências da FCM/UNICAMP; **Professor Doutor, assistente do Departamento de Neurologia da FCM/UNICAMP; ***Professor Doutor, assistente do Departamento de Cirurgia da FCM/UNICAMP; ****Professora Doutora, assistente do Departamento de Fisiologia da Faculdade de Medicina de Ribeirão Preto da Universidade de São Paulo SP; *****Médica, mestranda do curso de pós-graduação em Ciências Médicas da FCM/UNICAMP. Aceite: 20-novembro-1999.

Eliane A. Cintra - Rua Dr. Antônio de Souza Campos 35/31 - 13024-220 Campinas SP - Brasil. E.mail: licintra@obelix.unicamp.br 
Alterações neuropatológicas incluindo "swelling" cerebral difuso, hemorragias macroscópicas e graus variados de isquemia aguda distribuídas pelos hemisférios cerebrais, cerebelo e tronco encefálico, definidas in vivo como autólise, dependem do tempo em que o paciente permaneceu no respirador após o aparecimento de sinais clínicos e eletroencefalográficos da $\mathrm{ME}^{5}$. No entanto, apesar de a angiografia indicar infarto cerebral total, pequenas ilhas de córtex preservado foram demonstradas em pacientes que permaneceram sob ventilação mecânica por 3 a 4 dias $^{6}$. Ocorre também uma progressiva deterioração da função cardíaca na ME, provavelmente de causa multifatorial (déficit hormonal, aumento do metabolismo anaeróbico, diminuição das reservas de ATP). Um possível choque medular associado à herniação amigdaliana contribuiria para acentuar a queda da resistência vascular sistêmica ${ }^{7}$. Tal assunto tem conduzido vários investigadores a estudarem as alterações metabólicas, hemodinâmicas e hormonais em pacientes com ME ou em modelos animais experimentais ${ }^{3}$.

A ME dá origem a disfunção do centro vasomotor e a diminuição da liberação de catecolaminas, com consequente vasodilatação e diminuição da resistência vascular sistêmica. Isto, somado à desidratação secundária à poliuria e ao déficit de hormônio anti-diurético (arginina-vasopressina, AVP), determina hipovolemia e hipotensão, e esta é uma das alterações mais frequentemente relatadas. ${ }^{8}$

A ME resulta numa perda completa dos mecanismos centrais de regulação da estabilidade hemodinâmica mesmo em pacientes com suporte adequado de ventilação, correção hidroeletrolítica, adequação ácido-básica e suporte farmacológico convencional máximo da circulação. A hipertensão intracraniana leva a uma série de alterações neuropatológicas referidas como "tempestade adrenégica". No início, há um predomínio da atividade parassimpática, evidenciada por acentuada bradicardia, seguida de hiperatividade simpática que se manifesta por breve período de hipertensão e taquicardia que acompanham o processo de herniação do tronco cerebral, seguindo-se, então, o aparecimento abrupto ou insidioso de hipotensão ${ }^{9}$. Um dos fatores contribuintes para este distúrbio é o diabete insípido (DI), com diurese maciça, que ocorre em cerca de 38 a $87 \%$ dos casos..$^{10}$ Outro fator seria a disfunção do centro vasomotor, com consequente diminuição da liberação de catecolaminas, levando a queda da resistência vascular periférica e hipotensão. O DI é uma das alterações endócrinas da ME que ocorre devido a uma falência da síntese e/ou liberação de (AVP) no hipotálamo ou na hipófise posterior ${ }^{11}$.

A AVP é um hormônio peptídeo sintetizado nos núcleos supraópticos e paraventriculares do hipotálamo, sendo transportado à pituitária posterior onde é estocado. Este hormônio é liberado na circulação devido ao aumento da osmolaridade plasmática ou como uma resposta barorreflexa ao aumento do volume sanguíneo ou pressão arterial ${ }^{12}$. A AVP tem um papel importante na regulação do equilíbrio hídrico corporal através de sua ação antidiurética. Esta ação é mediada pelos receptores renais $\mathrm{V}_{2}$ ligados à adenilciclase, gerando AMP cíclico. A AVP também é capaz de causar vasoconstricção e aumentar a pressão arterial. Esta ação é mediada pelos receptores $V_{1}$ que, diferentemente dos $\mathrm{V}_{2}$, são ligados à fosforilase $\mathrm{C}$, aumentando a concentração intracelular do $\mathrm{Ca}^{++13}$. Transcorridas três horas após a ME, os níveis plamáticos de AVP ficam indetectáveis (inferiores a $0,1-0,5 \mathrm{pg} / \mathrm{ml}$ ), provocando um aumento da produção de urina hipoconcentrada ${ }^{14}$. Acredita-se que a diminuição de AVP circulante influencia de maneira preponderante a estabilidade cardiocirculatória destes pacientes que já apresentam uma insuficiência autonômica devido à ME.

No presente momento, não há consenso quanto ao melhor modo de manutenção da estabilidade hemodinâmica e preservação dos órgãos de pacientes em ME. Na prática, tem-se utilizado a reposição volêmica (cristalóides) em quantidades e velocidade de infusão suficientes para manter a pressão arterial sistólica acima de $100 \mathrm{mmHg}$ e a pressão venosa central ao redor de $12 \mathrm{mmHg}^{15}$. Uma vez conseguido o balanço hídrico correto, a persistência de hipotensão, apesar de uma pré carga ideal (PVC $12 \mathrm{~mm} / \mathrm{Hg}$ ), deverá ser tratada com fármacos inotrópicos. A dopamina é a droga mais empregada com esta finalidade. Doses de dopamina entre 1 e $5 \mu / \mathrm{kg} / \mathrm{min}$, atuando sobre os receptores dopa e beta adrenérgicos, favorecerão, ademais, a vasodilatação renal e esplâncnica, melhorando a perfusão destes órgãos. Doses maiores (>10 $\mu \mathrm{g} / \mathrm{kg} / \mathrm{min}$ ) produziriam, ao atuar sobre receptores alfa- 
adrenérgicos, uma progressiva vasoconstricção renal e sistêmica ${ }^{16}$. Por esta razão, fármacos com intensa ou exclusiva ação alfadrenérgica (adrenalina, noradrenalina, metoxamina e efedrina ) devem ser evitados para o controle da pressão arterial (PA) no potencial doador.

Scheinkestel et al. ${ }^{17}$ propõem, para crianças que ultrapassam a dose de noradrenalina de 0,5 $\mu / \mathrm{kg} / \mathrm{min}$, a administração de AVP para manter a pressão sanguínea. Em adultos, se há persistência da hipotensão na vigência do uso ótimo de drogas inotrópicas e esteróides, deve-se considerar esta conduta $^{18}$. Alguns autores orientam a utilização de análogos do hormônio anti-diurético para manter a estabilidade hemodinâmica destes pacientes ${ }^{14}$.

A ação da AVP é dose dependente. Em doses baixas (1-2 U/h ou 2-10 $\mu$ U/kg/min ), a atuação será sobre os receptores $\mathrm{V}_{2}$ das membranas celulares renais, aumentando a reabsorção de água e reduzindo a diurese. Em doses mais elevadas, seu efeito se dará sobre os receptores $V_{1}$ dos vasos sanguíneos produzindo hipertensão arterial, vasoconstricção do território pulmonar, mesentérico, hepático e coronariano, além da diminuição do fluxo sanguíneo renal sem alterar significativamente a diurese. ${ }^{19}$

Assim, considerando-se a atualidade e importância do tema, este estudo tem por objetivo discutir algumas análises experimentais e clínicas em relação ao comportamento da vasopressina na ME e seu papel na manutenção da estabilidade cardiocirculatória, bem como sua potencial utilidade no manuseio de pacientes que apresentam ME.

\section{ALTERAÇÕES HEMODINÂMICAS E COMPORTAMENTO DA VASOPRESSINA NA ME}

A vasopressina, dentro de uma faixa nanomolar, é um poderoso vasoconstrictor da musculatura lisa vascular, in vivo e in vitro. O efeito vasoconstrictor da AVP foi demonstrado in vitro, ao nível renal, aórtico, mesentérico, esplâncnico, músculo esquelético, artérias carótidas e arteríolas ${ }^{20,21}$.

Experimentos in vivo têm confirmado que aumentos fisiológicos de AVP no plasma são acompanhados de aumento da resistência vascular periférica, especialmente ao nível muscular esquelético, pele e território esplâncnico. Ademais, a AVP potencializa o efeito vasconstrictor das catecolaminas e da angiotensina II $^{22}$.

Em estudos prévios, ficou aparente que altas doses de AVP eram necessárias para aumentar a pressão arterial, doses estas muito maiores que a dose máxima necessária para a antidiurese. Por esta razão, considerouse que a ação pressora da AVP era mais farmacológica que fisiológica ${ }^{23,24}$. No entanto, outros estudos envolvendo mensurações hemodinâmicas em animais conscientes revelaram que sua ação pressora era significativamente subestimada ${ }^{25}$.

Na verdade, mostrou-se que a AVP é um potente vasoconstrictor, tanto quanto a noradrenalina ou a angiotensina II, e é capaz de aumentar a resistência vascular sistêmica em doses menores que a necessária para produzir concentração urinária máxima ${ }^{13}$. Apesar deste marcado efeito vasoconstrictor, a infusão de AVP em animais intactos ou em homens normais não aumenta significativamente a pressão arterial. No entanto, em situações de hipotensão, os barorreceptores cardíacos são hipersensibilizados. Esta ação é aparentemente mediada pelos receptores $\mathrm{V}_{1}$ da área postrema, uma área circunventricular localizada na medula oblonga. Isto causa um desvio à esquerda da curva barorreflexa frequência cardíaca-pressão arterial ${ }^{26,27}$. Esta ação explica o fenômeno bem documentado de que, para um dado aumento da pressão arterial, a AVP causa mais bradicardia do que outros vasoconstrictores. A remoção deste mecanismo tampão, através da denervação barorreceptora, aumenta enormemente a sensibilidade pressórica à $\mathrm{AVP}^{28}$. Esta hipersensibilidade pressórica também está aumentada em pacientes com insuficiência autonômica ${ }^{29}$.

A bradicardia que se segue à infusão de AVP também pode ser explicada devido ao seu efeito cronotrópico direto sobre o nó sinoatrial ou, ainda, por sua ação depressora sobre o miocárdio, direta ou secundária à vasoconstricção das artérias coronárias ${ }^{30}$. Em contrapartida ao tremendo efeito de estimulação do sistema barorreflexo, ocorre a supressão do sistema renina-angiotensina e redução da descarga adrenérgica simpática periférica e um aumento na excreção de sódio ${ }^{30}$.

Lagiewska et al. ${ }^{31}$ analisaram as alterações hemodinâmicas em 63 pacientes com morte encefálica (ME). De acordo com os distúrbios hemodinâmicos, os pacientes foram classificados em três grupos (A, B, C). Os pacientes do grupo A tinham pressão arterial sistólica (PAS) acima de $90 \mathrm{mmHg}$ com infusão de dopamina em 
doses < ou $=10 \mathrm{mg} / \mathrm{kg} / \mathrm{min}$ e débito urinário > que $0,5 \mathrm{ml} / \mathrm{kg} / \mathrm{h}$; nos pacientes do grupo B, a PAS era de 80 a 90 $\mathrm{mmHg}$ e estavam recebendo altas doses de dopamina (10 a $20 \mathrm{mg} / \mathrm{kg} / \mathrm{min})$ e os pacientes do grupo C eram pacientes com hipotensão progressiva em vigência do uso de altas doses de dopamina (> $20 \mathrm{mg} / \mathrm{kg} / \mathrm{min}$ ). Eles notaram que houve deterioração hemodinâmica progressiva, embora tenham sido administrados fluidos em todos os casos, e que estas alterações foram mais acentuadas em pacientes do grupo B e C. A deterioração foi manifestada pela necessidade de altas doses de dopamina $(15-25 \mathrm{mg} / \mathrm{kg} / \mathrm{min})$ nos pacientes destes grupos e $20 \%$ dos pacientes desenvolveram DI, sendo que a maioria destes eram dos grupos B e C.

Gramm et al..$^{32}$ analisaram a falência endócrina em 32 pacientes com ME. Vinte e cinco deles desenvolveram DI, sendo que em 12 destes pacientes isto ocorreu antes do início da ME. Em cinco pacientes deste estudo não houve evidência de DI até 48 horas após a ME, apesar dos valores de AVP permanecerem abaixo do normal. $\mathrm{O}$ motivo pelo qual somente $80 \%$ destes pacientes desenvolveram DI permanece obscuro. No entanto, os autores comentam que o efeito da deficiência não está restrito apenas aos distúrbios de osmolaridade e ao volume extracelular, pois Cowley, et al..$^{28}$ demonstraram que a AVP tem importante efeito amplificador direto sobre a pressão arterial em animais decapitados ou com denervação barorreceptora; ademais, o efeito pressórico da noradrenalina circulante foi consideravelmente aumentado pela AVP neste modelo. Möhring et al. ${ }^{29}$ demonstraram que indivíduos com atividade cardiorreflexa ausente ou diminuída têm resposta pressórica aumentada com níveis fisiológicos de AVP. Finalmente, os autores sugerem que condições similares poderiam ser aplicadas em um modelo experimental de ME.

Em outro estudo, Outwater \& Rockoff ${ }^{33}$ avaliaram 16 crianças com ME, das quais duas não desenvolveram DI. O que chama a atenção neste estudo é que das 14 crianças que desenvolveram DI, duas tiveram resolução espontânea dela, em uma, este fato ocorreu dentro de 24 horas após os achados clínicos de ME e, na outra, em 96 horas. Os autores colocam que a explicação da resolução do DI em pacientes com ME ainda é desconhecida, podendo ser devido a uma função residual do mesencéfalo ou à produção e liberação de hormônio anti-diurético em tecidos extracerebrais. Hupf et al. ${ }^{34}$, em um estudo experimental com coração de ratos, identificaram um RNAm da AVP e consequente indução da produção do peptídeo nestes corações isolados. Os mesmos autores localizaram a produção deste peptídeo em células endoteliais e vasculares da musculatura lisa das arteríolas, utilizando técnicas imunohistoquímicas, o que poderia explicar a hipótese de produção da AVP em tecidos extracerebrais.

Fiser et $\mathrm{al}^{35}$ avaliaram 34 crianças em ME. Os resultados revelaram que a DI ocorreu em $38 \%$ dos pacientes. O tempo da DI variou de 53 +/- 53h; $54 \%$ destes pacientes apresentaram taquicardia e $62 \%$ hipotensão. A hipotensão foi mais comum em pacientes com DI. Dois pacientes apresentaram resolução espontânea da DI e os autores atribuíram este fato à uma função diencefálica residual ou à produção e liberação extracraniana de hormônio anti-diurético. Os autores também fizeram uma avaliação anatomopatológica das pituitárias, sendo que algumas revelaram edema difuso, sem enfarte ou necrose, resultando em DI, que foi reversível em dois pacientes. A DI está presente em muitos, mas não em todos os pacientes com ME.

Sugimoto et al ${ }^{36}$ examinaram as alterações morfológicas e funcionais do sistema hipotálamo-pituitário em 28 pacientes com ME. Os níveis de hormônio anti-diurético tiveram queda dramática imediatamente após a ME, permanecendo, no entanto, detectáveis em alguns casos. As estruturas celulares do lobo posterior da pituitária (grânulos de vasopressina) permaneceram intactas. A vasopressina é sintetizada no hipotálamo sendo transportada à pituitária posterior pelos axônios, permanecendo estocada em grânulos. Estes autores identificaram estes grânulos de vasopressina mesmo após longo tempo de ocorrência da ME (20 dias), e parece ser esta a razão da permanência de níveis detectáveis de AVP em alguns pacientes nesta condição clínica. O hipotálamo, incluindo os núcleos supra-óptico e paraventricular, parece cessar sua função imediatamente após a ocorrência da ME. Assim, a secreção de AVP diminui rapidamente para níveis muito baixos, apesar da preservação das estruturas celulares da pituitária posterior ${ }^{36}$.

Mertes et al. ${ }^{37}$ investigaram, em modelo experimental, as alterações dos parâmetros hemodinâmicos e metabólicos em porcos com ME. Os parâmetros monitorizados foram: oferta de oxigênio $\left(\mathrm{DO}_{2}\right)$, consumo de oxigênio $\left(\mathrm{VO}_{2}\right)$, lactato sérico, pressão de capilar pulmonar (PCP), pressão de artéria pulmonar (PAP), pressão venosa central (PVC), débito cardíaco (DC), pressão arterial média (PAM) e dosagem de peptídeo natriurético atrial e vasopressina. Os animais foram divididos em dois grupos de dez e, em um dos grupos, a ME foi induzida. Os parâmetros hemodinâmicos e metabólicos foram monitorizados nos dois grupos antes da indução da ME e 5, 15, 30 e 60min após, seguindo-se controle de hora em hora até a sexta hora. Neste momento, foi administrado volume de expansão (solução salina, $20 \mathrm{ml} / \mathrm{kg}$ ) em ambos os grupos e os dados hemodinâmicos e metabólicos foram coletados. Os autores concluíram que a infusão rápida de volume a fim de manter a pressão arterial teve efeito deletério nos estados hemodinâmico e metabólico dos animais em ME, sugerindo que a infusão de volume 
com a finalidade de reduzir a utilização de agentes inotrópicos e vasoconstritores deve ser feita com cuidado no manuseio de potenciais doadores. Os níveis de vasopressina diminuíram nos dois grupos. No entanto, a diminuição foi mais acentuada nos animais com ME quando comparado com o grupo controle. Diante destes achados, os autores comentam que a queda dos níveis de vasopressina no grupo controle foi devida à administração de volume. Troppman \& Abrams ${ }^{38}$ contestam a conclusão de Mertes et al. ${ }^{37}$, sugerindo estudos adicionais para avaliar a utilização de volume, agentes inotrópicos, vasoconstrictores e reposição hormonal na manutenção de indivíduos com ME.

Estudos experimentais, utilizando um modelo canino, avaliaram as alterações endócrinas, metabólicas e hemodinâmicas que ocorrem na ME. O que se pôde observar nestes estudos foi o aumento dos níveis plasmáticos das catecolaminas 15 minutos após a ME: de $800 \%$ para a dopamina, de $700 \%$ para a noradrenalina e de $100 \%$ para a adrenalina ${ }^{3,39}$. Estes achados são compatíveis com os de outros estudos que utilizaram modelos similares de ME, em macacos, em que estes níveis se mantiveram altos por um período de 3 horas após a $\mathrm{ME}^{40}$. Também, Finkelsten, et al. ${ }^{41}$ já haviam relatado altos níveis de catecolaminas que diminuíram gradualmente durante período de duas horas após o início da ME. Os níveis de AVP e ACTH diminuíram e ficaram indetectáveis ao final dos experimentos; os níveis de cortisol, glucagon e insulina também diminuíram para níveis abaixo do controle ao final destes experimentos. Acidose metabólica ocorreu no início e no final dos experimentos. Isto foi atribuído, no início, a uma resposta hiperdinâmica e, no final, à perfusão tissular inadequada.

\section{UTILIZAÇÃO DA VASOPRESSINA NA MANUTENÇÃO DA ESTABILIDADE HEMODINÂMICA EM PACIENTES COM ME}

Num relato de estudo preliminar, Yoshioka et al. ${ }^{42}$, em 1986, conseguiram a manutenção da estabilidade circulatória a longo prazo pelo uso combinado de AVP e adrenalina em pacientes com ME. Em publicação posterior, o mesmo grupo ${ }^{43}$ estudou a efetividade do controle da estabilidade circulatória em pacientes com ME pelo uso combinado de AVP e catecolaminas. Vinte e cinco pacientes foram divididos em três grupos de estudo, a saber: Grupo I $(n=10)$; os pacientes receberam apenas adrenalina e na dose suficiente para manter a PA sistólica acima de $100 \mathrm{mmHg}$; Grupo II $(\mathrm{n}=2)$, receberam uma dose anti-diurética de $\operatorname{AVP}(0,1$ a $0,4 \mathrm{U} / \mathrm{h})$ associada à adrenalina como no Grupo I; e, Grupo III ( $\mathrm{n}=13)$, receberam uma infusão de AVP em dose pressórica (1 a 2 U/ h) associada à adrenalina. No Grupo I, a deterioração hemodinâmica progrediu rapidamente e a parada cardíaca ocorreu num prazo de $48 \mathrm{~h}$ após a ME $(24,1+/-17,2 \mathrm{~h})$. No Grupo II, o tempo de sobrevida foi de aproximadamente três dias ( $3,2+/-0,2$ dias) e a parada cardíaca não pôde ser evitada. No Grupo III, por outro lado, uma estabilidade cardiocirculatória foi mantida em todos os pacientes por tempo relativamente longo, enquanto durasse a infusão de AVP mais adrenalina (17,2+/-14,4 dias). Também, as necessidades de adrenalina no Grupo III foram diminuindo progressivamente com o tempo de infusão de AVP, tal o nível de estabilidade hemodinâmica alcançado. Os autores concluíram que a administração combinada de AVP em doses pressóricas e de uma calecolamina com a finalidade de estabilizar e manter adequada a circulação por um tempo prolongado, em pacientes com ME, é eficiente e confiável. Os mecanismos básicos desta ação, contudo, não são bem claros e necessitam elucidação.

Neste mesmo estudo ${ }^{43}$, os autores também investigaram o efeito da administração combinada de AVP e adrenalina. Cinco pacientes com estabilidade cardiocirculatória foram selecionados e randomizados para o estudo II. A hemodinâmica foi avaliada sob quatro condições (fases): Fase I, nem AVP nem adrenalina foram infundidas; Fase II, apenas AVP foi infundida; Fase III, a AVP foi retirada e apenas a adrenalina foi infundida; e, Fase IV, AVP e adrenalina foram infundidas. Em todas a fases, a avaliação foi realizada $60 \mathrm{~min}$ após a estabilização hemodinâmica dos pacientes. Os resultados deste estudo encontram-se resumidos na Tabela 1 (média +/- DP).

A análise dos efeitos hemodinâmicos advindos da combinação adrenalina-AVP demonstra que a adrenalina aumenta a PAM através do aumento do índice cardíaco (por aumento da contratilidade) devido ao seu efeito inotrópico direto sobre o coração, e a AVP aumenta a PAM acentuando a resistência vascular periférica por sua ação vasoconstrictora periférica. No entanto, se avaliarmos os dados, nem a adrenalina ou a AVP administradas separadamente são capazes de manter a estabilidade cardiocirculatória. Somente a administração combinada das duas pôde manter a estabilidade hemodinâmica por um longo tempo.

Estes autores também avaliaram, no mesmo estudo, a necessidade de utilização de catecolaminas para manter uma pressão arterial média acima de $90 \mathrm{mmHg}$. Quatro pacientes com estabilidade cardiocirculatória foram randomizados e em todos foi administrado AVP, na dosagem de $1 \mathrm{U} / \mathrm{h}$, adicionando-se adrenalina , noradrenalina e dopamina, sucessivamente.

As doses de noradrenalina necessárias para manter a PAM em torno de $90 \mathrm{mmHg}$ foram quatro vezes maiores que as de adrenalina. A noradrenalina têm pouco efeito sobre os receptores cardíacos, porém aumenta a 
Tabela 1. Efeitos da associação de vasopressina e adrenalina sobre os parâmetros cardiocirculatórios. Adaptado de Iwai et al.

\begin{tabular}{cccccc}
\hline Fase & PAM & IC & IRVP & PCP & mVcf \\
\hline I & $49,6(6,7)$ & $3,93(1,03)$ & $848(137)$ & $11,1(3,1)$ & $1,16(0,33)$ \\
II & $61,0(9,3)^{\mathrm{a}}$ & $3,64(0,88)$ & $1157(214)^{\mathrm{b}}$ & $11,4(3,6)$ & $1,05(0,20)$ \\
III & $67,4(15,3)^{\mathrm{b}}$ & $4,52(0,79)^{\mathrm{b}}$ & $1020(219)$ & $12,3(2,9)^{\mathrm{b}}$ & $1,41(0,27)^{\mathrm{b}}$ \\
IV & $101,0(15,1)^{\mathrm{c}}$ & $4,86(1,36)^{\mathrm{a}}$ & $1558(461)^{\mathrm{b}}$ & $14,8(4,6)^{\mathrm{b}}$ & $1,32(0,23)$ \\
\hline
\end{tabular}

Fase I, nenhuma droga foi infundida; Fase II, somente vasopressina foi infundida; Fase III, apenas adrenalina foi infundida; Fase IV, adrenalina e vasopressina foram infundidas conjuntamente. PAM, pressão arterial média (mmHg); IC, índice cardíaco, (1/ $\left.\mathrm{min} / \mathrm{m}^{2}\right)$; IRVP, índice de resistência vascular periférica, (dyna/seg/ $\left.\mathrm{cm}^{3}\right)$; PCP, pressão de capilar pulmonar, $(\mathrm{mmHg}) ; \mathrm{mVcf}$, velocidade média de encurtamento circunferencial da fibra cardíaca (circ./seg). ${ }^{\mathrm{a}} \mathrm{p}<0,05 ;{ }^{\mathrm{b}} \mathrm{p}<0,01 ;{ }^{\mathrm{c}} \mathrm{p}<0,001$.

PAM devido ao seu efeito sobre os receptores alfa-periféricos aumentando a resistência vascular. A dopamina, em contraste, aumenta a PAM devido a um acentuado efeito sobre o índice cardíaco, por aumento da frequência cardíaca e da velocidade de contração, demonstrando efeitos inotrópico e cronotrópico positivos. A adrenalina aumenta a PAM devido aos fatores citados acima. No entanto, comparada à dopamina, a adrenalina tem pouco efeito sobre a frequência cardíaca. Este estudo confirmou que a AVP é agente importante para manter a estabilidade cardiocirculatória em pacientes com ME. A dose antidiurética, no entanto, não é suficiente para isso. A dose pressora parece ser essencial. Quando combinada com outras catecolaminas, dentre todas, a adrenalina parece produzir uma estabilidade cardiocirculatória através de um mecanismo de melhora dos principais efeitos de cada uma: a AVP através do efeito vasoconstrictor e a adrenalina com seu efeito inotrópico. Além disso, as doses de adrenalina necessárias foram menores que as de noradrenalina, e a dopamina aumentou acentuadamente a frequência cardíaca ${ }^{43}$.

\section{CONCLUSÃO}

A morte encefálica dá origem a uma série de distúrbios autonômicos, metabólicos e hemodinâmicos. Tais distúrbios levam a deterioração da estabilidade cardiocirculatória e da perfusão tissular, culminando com a parada cardíaca. A vasopressina parece ter papel importante na manutenção desta estabilidade, interferindo na perfusão tissular e, consequentemente, evitando a parada cardiocirculatória. No entanto, o comportamento da vasopressina na morte encefálica e os seus efeitos ainda não estão muito claros, necessitando de melhor elucidação através de futuras investigações.

\section{REFERÊNCIAS}

1. Nelson RF. Determination of brain death. Guidelines for the diagnosis of brain death. Can J Neurol Sci 1986;13:355-358.

2. Balslev-Jorgensen P, Heilbrun MP, Boysen G, Rosenklint A, Jorgensen EO. Cerebral perfusion correlated with regional cerebral blood flow, EEG and aorta / cervical arteriography in patients with severe brain disorders progressing to brain death. Cerebral blood flow and intracranial pressure. Proc $5^{\text {th }}$ InternatSymposium. Rome, 1971:111 Eur Neurol 1972;8:207-272.

3. Bittner HB, Kendal SWH, Chen EP, Craig D, Trigt PV. Endocrine alterations and metabolic responses in a validated canine brain death model. J Crit Care 1995;10:56-63.

4. Bucheler E, Kaufer C, Dux A. Zerebrate angiographic zur bestimmung des hirtudes. Fortschr Roentgenstr 1970;113:278-296.

5. Walker AE, Diamond EL, Moseley J. The neuropathological findings in irreversible coma. J Neuropath Exp Neurol 1975;34:295-323.

6. Ingvar DA, Widen L, Hjarnndo D. Sammenfatthing Au ett Symposium. Lakartidningen 1972;69:3804-3814.

7. Robertson KM, Cook OR. Perioperative management of the multiorgan donor. Anesth Analg 1990;70:546-556.

8. Soifer BE, Gelb AW. The multiple organ donor: identification and management. Ann Intern Med 1989;110:814-823.

9. Cooper DKC, Novitzky D, Wicomb WB. Pathophysioloy of brain death in the experimental animal: extracranial aspects. Transplant Proc 1998;30(Suppl. 7):25-28.

10. Lindop MJ. Basic principles of donor management for multiorgan removal. Transplant Proc 1991;23:2463-2464.

11. Marks JR. Central diabetes insipidus. In Levin DL, Morris RC, Moore GC (eds). A pratical guide to pediatric intensive care. St Louis: Mosby, 1979:222-223.

12. Schrier RN, Berl T, Andersen RJ. Osmotic and nonosmotic control of vasopressin release. Am J Physiol 1979;236: 321-332.

13. Reid IA, Schwartz I. Role of vasopressin in the control of blood pressure. In Martini F, Ganong WF (eds). Frontiers in neuroendocrinology. New York: Raven Press, 1984:171-197. 
14. Valero R, Cabrer CA, Manyalich M, García-Fagès, LC. Mantenimiento del donante de órganos. Rev Esp Anestesiol Reanim 1992;39:293-300.

15. Randel T, Orko R, Hockerstot K. Perioperative fluid management of the brain dead multiorgan donor. Acta Anestesiol Scand 1990;34:592-595.

16. Goldberg LI. Dopamine: clinical uses of an endogenous catecholamine. N Engl J Med 1974;291: 707-710.

17. Scheinkestel CD, Tuxen DV, Cooper DJ, Boot W. Medical management of the (potential) organ donor. Anaesth Intens Care 1995;23:51-59.

18. KinoshitaY, Okamoto K, Yahata K, Yoshioka T, Sugimoto H. Clinical and pathological changes of the heart in brain death maintained with vasopressin and epinephrine. Pathol Res Pract 1990;186:173.

19. Jackson EK. Vasopressin and other agents affecting the renal conservation of water. In Hardman JG, Limbird LE, Gilman AG (eds).Goodman \& Gilman's pharmacological basis of therapeutics 9Ed, New York: McGraw Hill, 1996:715-731.

20. Altura B, Altura B. Vascular smooth muscle and neurohyphophyseal hormones. Fed Proc 1977;36:1853-1860.

21. Altura B, Altura B. Actions of vasopressin, oxytocin and synthetic analogues in vascular smooth muscle. Fed Proc 1984;43:80-86.

22. Bartelstone HS \& Nasmyth PA. Vasopressin potentiation of catecholamines actions in dog, cat and rat aortic strips. Am J Physiol 1965;208:754-759.

23. Landry DW, Levin HR, Gallant EM, et al.. Vasopressin deficiency contributes to the vasodilation of septic shock. Circulation 1997;95:1122-1125.

24. Reid IA. Role of vasopressin deficiency in the vasodilation of septic shock. [Editorial]. Circulation 1997;95:1108-1110.

25. Montani JP, Liard JF, Schoun J, Möhring J. Hemodynamic effect of exogenous and endogenous vasopressin at low plasma concentration in conscious dog. Circ Res 1980;47:346-355.

26. Luk J, Wong V, Wong J, Chang D, Choo L; Reid EA. Role of $\mathrm{V}_{1}$ receptors in the action of vasopressin on the baroreflex control of the heart. Am J Physiol 1993;265:524-529.

27. Undesser KP, Hasser EM, Haywood JR, Johnson AK, Bishop HS. Interactions of vasopressin with the area postrema in arterial baroreflex functions in conscious rabbits. Circ Res 1985;56:410-417.

28. Cowley AW Jr, Monos E, Guyton AC. Interactions of vasopressin and baroreceptor reflex system in the regulation of arterial of blood pressure in the dog. Circ Res 1974;34:505-514.

29. Möhring J, Glanzer K, Maciel JA Jr, et al.. Greatly enhanced pressor response to antidiuretic hormone in patients with impaired cardiovascular reflexes due to idiophatic orthostatic hypotension. J Cardiovasc Pharmacol 1980;2:367-376.

30. Johnston CI: Vasopressin in circulatory control and hypertension. J Hypertension 1985;3:557-569.

31. Lagiewska B, Pacholczyk M, Walaszewski J, Rowinski W. Hemodynamic and metabolic disturbances observed in braindead organ donors. Transplant Proc 1996;28:165-166.

32. Gramm HJ, Meinhold H, Bickel U, et al.. Active endocrine failure after brain death. Transplantation 1992;54:851-857.

33. Outwater KM, Rockoff A. Diabetes insipidus accompanying brain death in children. Neurology 1984;34:1243-1246.

34. Hupf H, Grimm D, Riegger AJG, Schunkert H. Evidence for vasopressin system in the rat heart. Circ Res 1999;9:365-370.

35. Fiser DH, Jimenez JF, Wrape V, Woody R. Diabetes insipidus in children with brain death. Crit Care Med 1987;15:551-553.

36. Sugimoto T, Sakano Y, Kinoshita M, Yoshioka T. Morphological and functional alterations of the hypothalamic-pituitary system in brain death with long term bodily living. Acta Neurochir (Wien) 1992;115:115-131.

37. Mertes MP, El Abassi K, Jaboin Y, et al.. Changes in hemodynamic and metabolic parameters following induced brain death in the pig. Transplantation 1994;58:414-418.

38. Troppman C, Abrams HG. Parameters in brain dead pigs. Transplantation 1995;60:111-114.

39. Chen EP, Bittner HB, Kendall SWH, Vantrigt P. Hormonal and hemodynamic changes in a validated animal model of brain death. Crit Care Med 1996;24:1352-1359.

40. Novitzky D, Cooper DKC, Wicomb WN. Electrocardiographic, hemodynamic, and endocrine changes occurring during experimental brain death in the Chacma baboon. J Heart Transplant 1984;4:63-69.

41. Finkelstein I, Toledo-Pereyra LH, Castellanos J. Physiologic and hormonal changes in experimentally induced brain dead dogs. Transplant Proc 1987;5:4156-4158.

42. Yoshioka T, Sugimoto H, Uenishi M, et al.. Prolonged hemodynamic mantainance by the combined administration of vasopressin and epinephrine in brain death: a clinical study. Neurosurgery 1986;18:565-567.

43. Iwai A, Sakano T, Uenishi M, Sugimoto H, Yoshioka T, Sugimoto T. Effects of vasopressin and catecholamines on the maintenance of circulatory stability in brain-dead patients. Transplantation 1989; 48:613-617. 